\title{
Remote Android Assistant with Global Positioning System Tracking
}

\author{
Jyothi T. S., Catherine Mathew, Irene George \\ Department of Computer Science and Engineering, Jyothi Engineering College, Cheruthuruthy
}

\begin{abstract}
The paper describes a method which binds a web application and an Android mobile application, to provide remote exploration services. A remote exploration system has been developed using web server, database, and an Android mobile phone which supports GPS functionality. Web server deals with user interaction part which consists of setting command signals through which user can interact with remote devices. The system is loosely coupled with the introduction of an intermediate server. The user sends commands through browser which maps to the server. Mobile application is responsible for executing user commands. The Web application interacts with the server, fetches commands, executes and provides appropriate responses. The command is sent from the web page. The Android mobile phone reads the sent command and performs predefined action. It can also send GPS location of the mobile phone to server's database.
\end{abstract}

Keywords: GPS, Android mobile phone, Web application, Contacts, Call log, SMS log.

\section{Introduction}

The paper helps to access an Android mobile remotely. We can access and retrieve data from our mobile phone even if it is not next to us. [4] The concept of remote assistance is utilized here. The data which could be fetched from the phone includes contacts, call logs, SMS logs, and current location of the mobile. The user can access and perform operations through a Java platform. The web application is made to run in the end system which acts as the server and the Android phone acts as the client.

GPS is a Global Positioning System based on satellite technology [5] which provides location and time information in all weather conditions, anywhere on or near the Earth where there is an unobstructed line of sight to four or more GPS satellites. It helps to find the precise location of a person, or that of an asset to which it is attached and to record the position of the asset at regular intervals of time. The recorded location data is projected onto Google Map to ease users in viewing the tracked location. This technique allows us to find the phone's location.

Android is an open-source Linux-based operating system designed mainly for smart phones and tablets. It is maintained as an open source project by Google. [1] This open source code and licensing allows the developers and also the device manufacturers to modify the software according to their needs. Android platform has brought about infinite technologies in application development. Android is mainly based on ARM architecture platform [3]. A mash up combines two or more services to create an app. You can create a mash up by using the camera and Android's location services. The Android Market puts your app right into your users' hands easily. Users don't have to go searching the Internet to find an app to install. They just simply go to the Android Market that is preinstalled on their device, and they have access to all your apps. The user can access and perform operations through a Java platform. The web application is executed in the end system which acts as the server and the Android phone acts as the client. Commands are sent from the application and these commands are read by the Android phone when it is active, executed, and suitable outcomes are generated. The Android operating system is open platform, meaning that it's not tied to one hardware manufacturer and/or one provider [6].

\section{[A] CLIENT SIDE WEB APPLICATION}

\section{System Operation}

Client part is visible to the end user and users interact with it by sending commands and receiving the response due to execution of command. The client side includes the web application, which is been accessed through the web browser. The web application, opens with the login page. The users who have already registered can directly enter into the home page by logging in. New users need to register initially after which they can log in to the home page. Once logged in, they are directed to the home page consisting of different buttons, representing the appropriate commands to be performed in the Android phone. Results are fetched from the database. Many users can be connected to the server simultaneously.

\section{[B] SERVER SIDE WEB APPLICATION}

The server side part processes and handles requests from the client side web application and remote android device application. For instance, server receives request from the user and stores commands to the 
mobile application on a regular basis. The Android phone regularly pings the database to check whether new commands has arrived. If so, the server side gives the command to the Android side, where they are executed and the results are provided to the database and finally stored there. The connection is confirmed with authentication. The main functionalities involved in the proposed system are access to call logs, retrieval of messages, locating of phone and listing of contacts. Apache Tomcat was used as the web server for managing the web application.

\section{[C] ANDROID APPLICATION}

Android has a couple of Java core library and it has added additional libraries to provide support for development of the android application. Android mobile application is responsible for executing stored commands. For developing this Android application, Android SDK and Eclipse were used. A comprehensive set of development tools is included in the Android software development kit (SDK). Eclipse being the officially supported integrated development environment (IDE) uses the Android Development Tools (ADT) Plug-in for Android development.

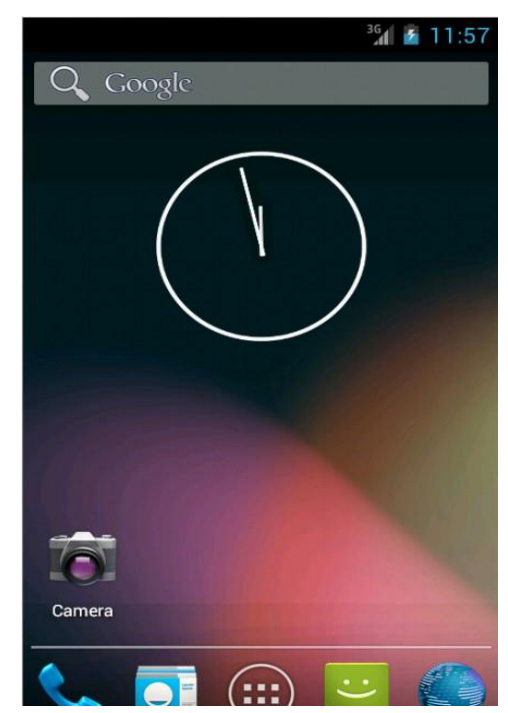

Figure 1 Emulator

The emulator shown in figure 1 is used while running the app in a local host using Apache Tomcat.

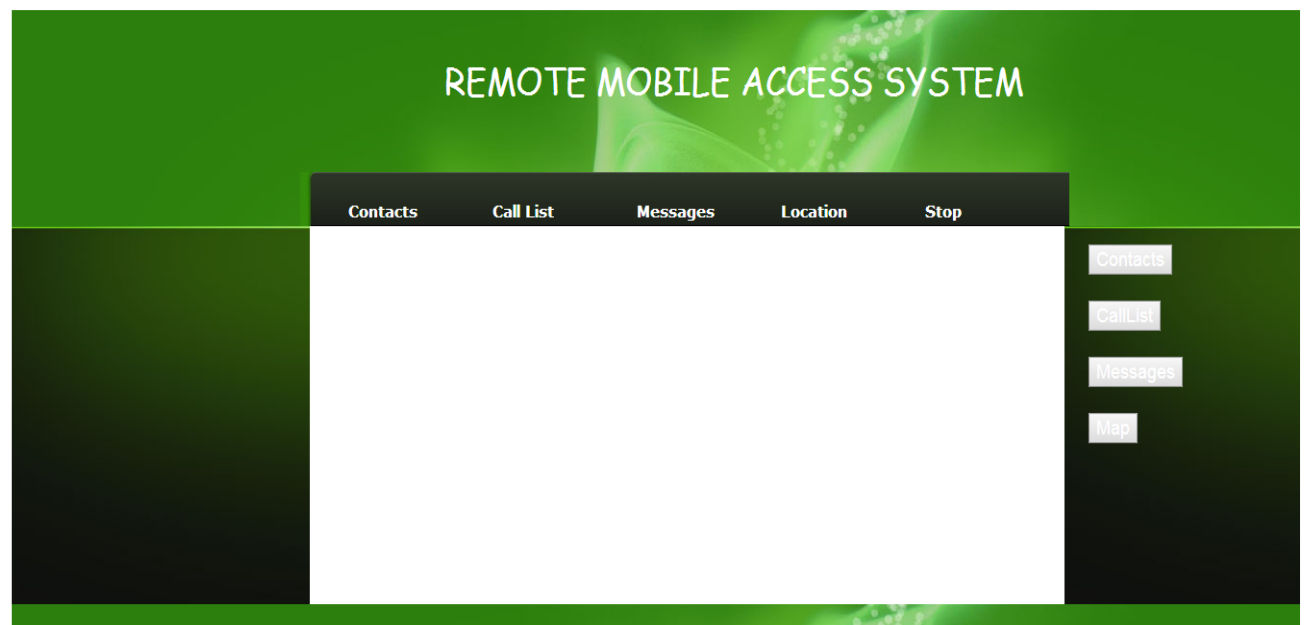

Figure 2 Home page

Figure 2 shows the home page of our web application.

\section{CONTACT}

In this paper, you may just want to add an easy way for the users to contact you from an app. This is all possible with the built-in APIs. The app can access user contacts that are stored on the phone by giving 
permission. You can use this feature to display the contacts in a new or different way. Maybe you don't like the built-in Contacts application. With the ability to access the contacts stored on the phone, nothing is stopping you from writing your own.

\section{CALL LOG}

The list of all calls i.e. missed calls, received calls and dialed calls can be retrieved from the phone. On executing this command, the log containing the contact's name and number will be displayed.

\section{MESSAGE}

The Android Cloud to Device Messaging framework allows you to send a notification from your Web server to your app and vice versa. You can view all the messages or the message of a particular contact. On executing this command, the log containing the contact's name and message will be displayed.

\section{LOCATION}

The Android OS combined with a phone's GPS allows developers to access a user's location at any given moment within a distance. The figure 3 , shows the output containing the latitude and longitude of the phone's current position.

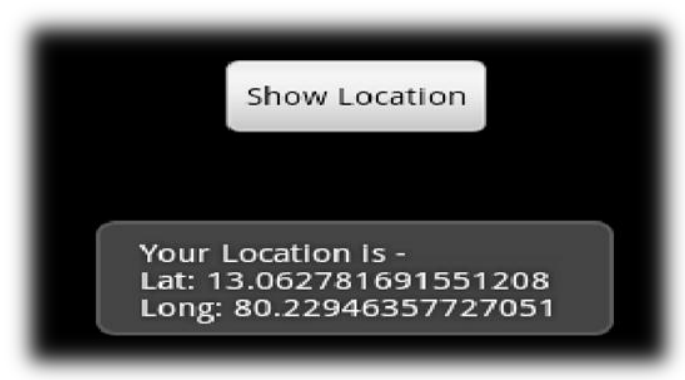

Figure 3 GPS Location

\section{Software requirements:}

\section{System Design}

> Android SDK - Provides access to Android in-built libraries and allows you to develop for Android. The Android SDK is composed of a debugger, Android libraries, a device emulator, documentation. [2] This is inevitable during development phase.

$>$ Eclipse IDE (integrated development environment) - Puts together Java, the Android SDK, and the Android ADT (Android Development Tools), and provides tools for developer to write Android programs. Developed using Java, the Eclipse platform can be used to develop rich client applications, integrated development environments and other tools. Eclipse can be used as an IDE for any programming language for which a plug-in is available.

$>$ Android ADT - Does a lot of the grunt work for developer, such as creating the files and structure required for an Android app. ADT adds functionality to Eclipse to do a lot of the work for developer. The ADT allows developer to create new Android projects easily; it creates all the necessary base files so that developer can start coding your application quickly. It also allows developer to debug their application using the Android SDK tools. Finally, it allows them to export a signed application file, known as an Android Package (APK), right from Eclipse, eliminating the need for some command-line tools.

$>$ Apache Tomcat - It is a web application server. Since the web pages are written in JSP, they are stored in the webapps directory in the form of a compressed file called a WAR file. This file is executed using the server's help in a browser.

The figure 4 below shows the diagrammatic representation of the working of this paper. 


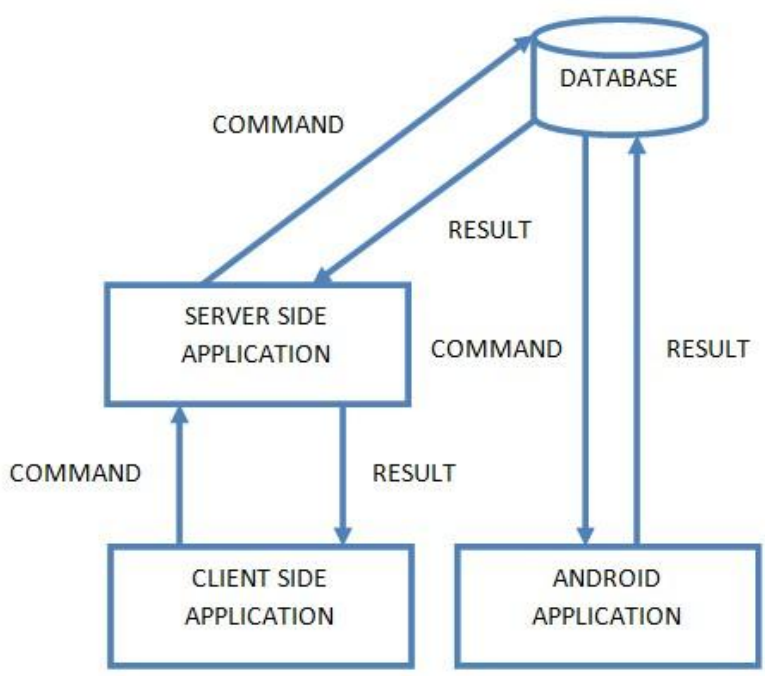

Figure 4 Working model

Flowchart:

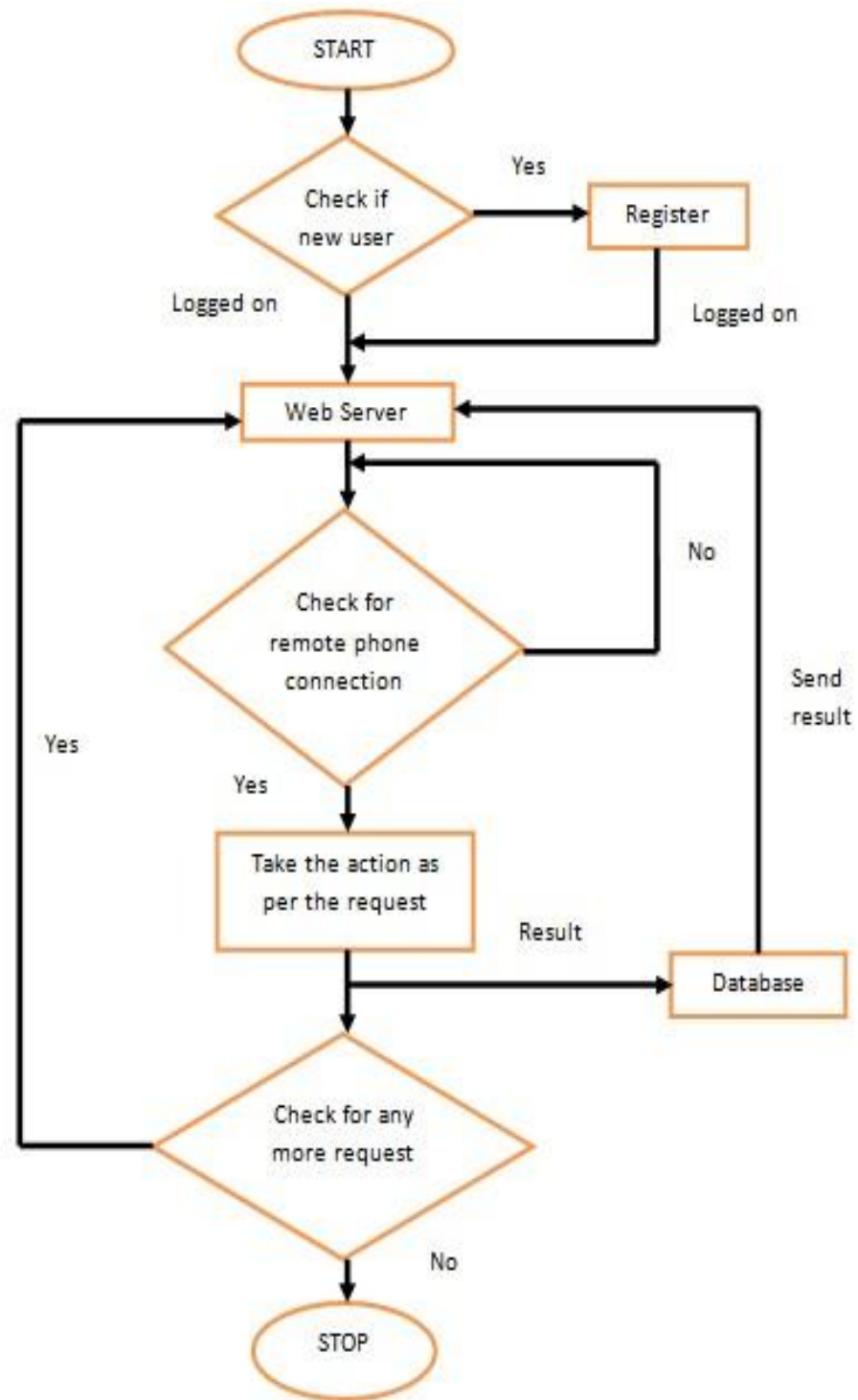

Figure 5 
The flowchart in figure 5 shows the working of the system. This working can be summarized in the following manner.

Web Side:

1. New users have to register by providing username, password and mobile number

2. User logins with username and password

3. Selection of desired option from the home page

This can be done by clicking on the appropriate button for the retrieval of messages, call logs, location or contacts.

4. Execution of commands Command is sent to Android device

5. Fetch and save the response in the database

6. Stop service

Mobile Side:

1. Download the apk file in the device

2. Install the apk file

3. Open and run file in background

\section{Conclusion}

We have designed an application to access a remote Android phone from web server. From the various related works and the base paper, we have drawn a blueprint of the application. The convenience of having access to critical information by using a network access infrastructure can be made easier through a wireless connection between Android phone and server connected to the internet. The proposed system has an application module framework of tracking GPS technology for effective information delivery and management. For location tracking we are using Geographical Positioning System. The raw data provided by GPS receiver is captured by the software and processed to extract the required location. Our proposed architecture offers a very promising solution for organizations which are in need of a secure flexible and cost-effective remote access methodology using Android phone.

\section{Reference}

[1] William Enck, Machigar Ongtang, Patrick McDaniel, Understanding Android Security, IEEE Computer Society, IEEE Security \& Privacy, January/February 2009

[2] William Enck, Damien Octeau, Patrick McDaniel, Swarat Chaudhuri, A Study of Android Application Security, Systems and Internet Infrastructure Security Laboratory, Department of Computer Science and Engineering, The Pennsylvania State University

[3] Jaya Bharathi, Srinivasa Rao, Remote computer access through Android mobiles, IJCSI International Journal of Computer Science Issues, Vol. 9, Issue 5, No 3, September 2012, ISSN (Online): 1694-0814

[4] E. Tomur, R. Deregozu, T. Genc, A Wireless Secure Remote Access Architecture Implementing Role Based Access Control: WiSeR, World Academy of Science, Engineering and Technology 182008

[5] B. P. S. Sahoo, Satyajit Rath, INTEGRATING GPS, GSM AND CELLULAR PHONE FOR LOCATION TRACKING AND MONITORING, Proceedings of Geomatrix'12, India

[6] Md. Ashraful Alam Milton, Ainul Anam Shahjamal Khan, Web Based Remote Exploration and Control System Using Android Mobile Phone, IEEE/OSA/IAPR International Conference on Informatics, Electronics \& Vision, 2012 